\title{
A Study on the Design of Rural Material Flow System Based on the Traditional Chinese Twenty-four Solar Terms - A Case Study of Lifengjie in Liyang City, Jiangsu Province, China
}

\author{
Xiao-Yu Xie ${ }^{1, a}$, Sheng-Xi Fan ${ }^{2}$ \\ ${ }^{1}$ Behavior Cognition Design Research Lab, Tongji University, No 1239 Si Ping Road, Shanghai, China \\ ${ }^{2}$ Behavior Cognition Design Research Lab, Tongji University, No 1239 Si Ping Road, Shanghai, China
}

\begin{abstract}
In the view of Chinese traditional twenty-four solar terms, the circulation of various substances in the material flow system of base production and life is systematically analyzed, and on the basis of the existing system problem point, as well as by combining with the concept of the twenty-four solar terms and the system design method, the author reconstructs each subsystem of the base material flow system, moreover, the author eventually connects the subsystems and reconstructs a material flow system conforming to the traditional Chinese solar terms and conforming to the requirements of ecological sustainability.
\end{abstract}

\section{Introduction}

Limit to Growth describes such a scene: the huge explosion in population, environmental degradation, industrial growth without restraint, and the loss of urban morality, causing the loss of earth's resources as well as environmental burdens in human economic activities, eventually leading to the environmental disruption [1]. Agricultural production is governed by the laws of industrial production, whose subversion of the laws of nature can affect the whole food production system, and such a single development model will have more serious environmental and social problems for developing countries, or less developed areas, thus causing immeasurable damage to the traditional customs and cultures that have been brought together in the most harmonious way with the ecosystem for hundreds of years [2]. The return of the twenty-four solar terms and the contemporary people's concern about the safety of diet are the background of this study, while because of its better preservation of the characteristics of Chinese traditional farming society, Lifengjie has become the experimental field for systematic design and research based on the traditional twenty-four solar terms culture in China. The understanding and the evaluation of the environmental, social and economic performances of complex agricultural food systems is probably the real challenge, and the design of more sustainable alternatives has been recognized as necessary for a correct territorial management [3]. However, the system design started late in China and lacked practice cases, so if we can use systematic design method to carry on systematic research to "twenty-four solar terms", and carry on the practice of system design, on the one hand, it provides a new idea for the study of the twenty-four solar terms,

\footnotetext{
${ }^{a}$ Corresponding author: xiexiaoyu_tongji@163.com
} 
and on the other hand, it fills the gaps of the study of the traditional agricultural circulation model of the Orient in the design of the system, especially the ecological design.

\section{Tools and Methods}

System design is an applied design method combining system thinking and industrial design. In this study, the author uses the system design method to analyze the elements of the base material flow system [4].

\subsection{Application range of system design}

As a subject, design has both sensibility and rationality, and is widely used in art, anthropology, economics and management, technological progress and other aspects. And the system design is concerned with human development and economic benefits, and reflects the significance and value of design, moreover, it differs from artistic design from the inner emotional level, and requires a certain amount of scientific measurement and analysis to solve the problem rationally. Their relationship is shown in the following figure. 1:

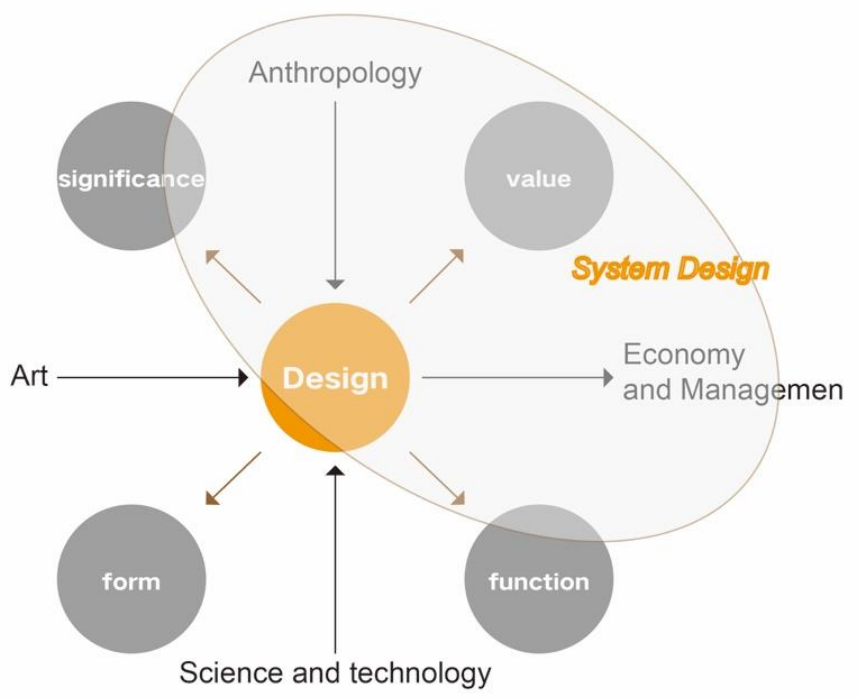

Figure 1. Range of application system design

\subsection{System design method}

Systematic boundaries should be delineated at the beginning of systematic studies, the qualitative and quantitative methods can be used to study the nature, definition and quantity of the input and output in the system according to the resources in the system boundary, moreover, the relationship between the activities in the system should also be paid attention to. At the same time, we should pay attention to the factors such as the ability of the participants and the action ability of the system, which will affect the efficiency of the system [4]. The relationship is Showed in figure. 2. 


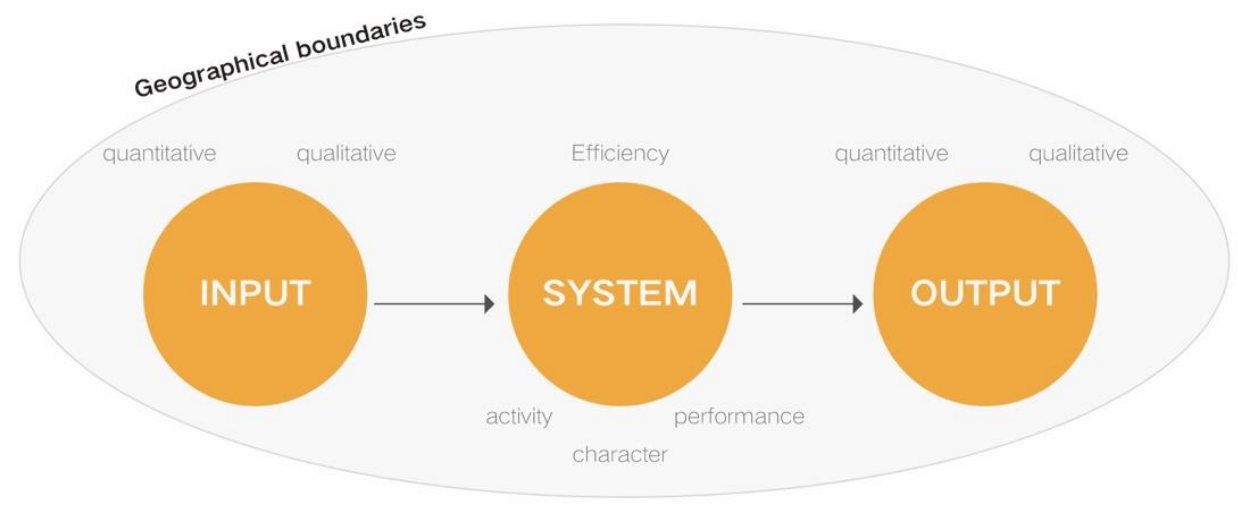

Figure 2. System design method

\subsection{Guidelines for system design}

\subsubsection{Waste is resources}

Mechanical linear thinking often results in excessive exploitation of resources and the production of large amounts of waste, while the rational planning system can improve the efficiency of the system, thereby enabling the output of a system to be transformed into another system's resources, then there will be no waste.

\subsubsection{Open system}

Various systems in nature, including objects as small a enzyme catalysis in cells, as well as the energy exchange between the earth and the universe, are all open systems [4]. The system design is an open system that allows material flow and energy flow to flow together, which is different from closed loop systems in industrial ecological design.

\subsubsection{The original system is related to other systems}

The open system repeats the functions of the system in dynamic equilibrium, while maintaining itself and evolving with other systems associated with it, and when an element of a system changes, the system adjusts itself, thereby affecting the output of the system, and the change of the output of this system will inevitably affect the input of another system [5]. Finally, each system is connected with each other and adjusted dynamically.

\subsubsection{Geographical importance}

The system has boundaries which can be physical boundaries, such as cell walls; and they can be cultural boundaries as well, such as Islamic cultural circle, etc., and the system is meaningful only if it is discussed within a certain boundary. Different geographical environment contains different resources, and breeds unique resources, culture and residents. Therefore, the emphasis on regional differences is the basis of system design, so there are no two identical system designs.

\subsubsection{The importance of man in the system}


The system has boundaries which can be physical boundaries, such as cell walls; and they can be cultural boundaries as well, such as Islamic cultural circle, etc., and the system is meaningful only if it is discussed within a certain boundary. Different geographical environment contains different resources, and breeds unique resources, culture and residents. Therefore, the emphasis on regional differences is the basis of system design, so there are no two identical system designs.

The idea of suiting measures to local conditions and waste utilization advocated by the system design coincides with the concept of fine agricultural production in the traditional twenty-four solar terms of China, in addition, the ecological farming experience, vegetable rotation for sericulture reeling in Chinese traditional agriculture is also praised by the scholars who use the system design method to carry on the circulation agriculture pattern research, and is often listed as a positive case in various books. Because of this, the system thinking and system design approach can work when we try to optimize the life system of the project base according to the twenty-four solar terms. The idea of suiting measures to local conditions and waste utilization advocated by the system design coincides with the concept of fine agricultural production in the traditional twenty-four solar terms of China, in addition, the ecological farming experience, vegetable rotation for sericulture reeling in Chinese traditional agriculture is also praised by the scholars who use the system design method to carry on the circulation agriculture pattern research, and is often listed as a positive case in various books[2]. Because of this, the system thinking and system design approach can work when we try to optimize the life system of the project base according to the twenty-four solar terms.

\section{Achievements}

\subsection{Linear}

In administrative relations, Lifengjie belongs to Pingqiao village, Tianmu Lake Town, Liyang City, which has 103 households, more than 300 resident population, and whose north-south length is about $1000 \mathrm{~m}$, east-west span is about 500 meters, the lowest point is 100 meters, and the hills are around 230 meters above sea level. The total area of cultivated land is $279 \mathrm{mu}$, of which 143 are paddy fields and 145 are dry land. The bamboo forests around the mountains are about $900 \mathrm{mu}$, and the area of the ponds is $23 \mathrm{mu}$. Moreover, agriculture and forestry are the main economic fields, paddy fields mainly produce two season rice, dry land grows various kinds of vegetables and chestnuts and other economic crops; and the forest provides bamboo shoots, bamboo shoots, bamboo and other raw materials, therefore, it maintains the production and life style of the family units in the traditional farming society of China.

Lifengjie is surrounded by the Lifeng Mountain to form natural geographical boundaries, therefore, the author's research will also take this as the research boundary. The villagers there open up the flat areas in the middle of the depression into their fields, and their residential areas are located on the foothills of the depressions, thus their main sources of water resources are natural rainfall and mountain spring water, and villagers set up several ponds to function as water storage. In addition, according to the different types of land, the main output areas of various ingredients are also reflected in the Figure. 3.

Based on the analysis of the material flow direction, rhythm and economic benefits of the existing life production system, the author sums up the systematic characteristics and problems of the current material flow in Lifengjie: 


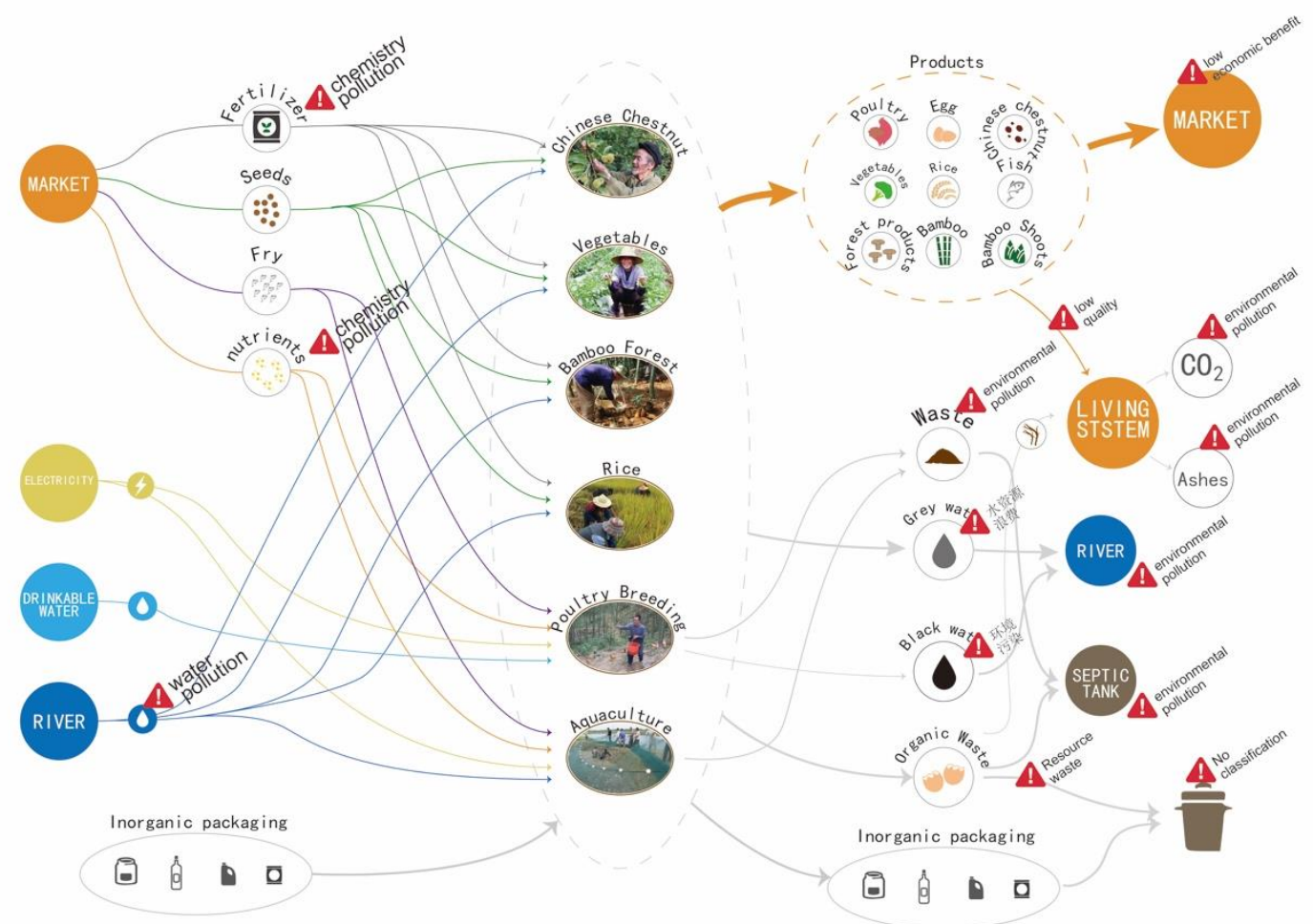

Figure 3. Linear agriculture system and problem points

\subsubsection{The whole is in line with the twenty-four solar terms}

Lifengjie keeps many production experiences of Chinese traditional agriculture in agricultural production, such as plant crops in rotation, and raise chickens in bamboo forests, etc.. In selecting crop types, in addition to the economic benefits, seasonal crop suitability is also considered, therefore, it has done better in accordance with local conditions, moreover, it makes full use of local weather, light and heat resources, and because bamboo is suitable for production in this area, it energetically develops the related industries of bamboo forest industry.

\subsubsection{The diet of the residents does not meet the requirements of the solar terms}

After systematic analysis, there are two main sources of food ingredients for residents in Lifengjie: First of all, the ingredients come from stores in Pingqiao village, which include all kinds of anti season foods, vacuum canned food and so on. These foods share a common feature: after being preserved in the processing area, they are transported to Pingqiao village, and then reached the villagers' hands through the stores. And the ingredients are the products of over industrialized production, which are co opposed by two authors in Slow Food and Blue Economy. Whether GM crops affect the body is not yet conclusive, however, the damage done to humans by a large amount of chemical agents have been paid attention to by an increasing number of people, what's more, longdistance transportation often destroys the nutrition of the ingredients, therefore, the author believes that the quality of such ingredients is not high. The other source of food ingredients for residents in Lifengjie is local product, and this approach seems to be much better in quality than the above approach, however, when the author systematically analyze, it is found that a large number of local crops are produced by using chemical fertilizers and chemical herbicides, insecticides and so on, and 
these chemicals remain on the surface of the crop and enter the soil and water and enter the crops, so the ingredients are not of high quality.

\subsubsection{The waste of part resources}

When we systematically analyze the production process of its ingredients, we can find another point of pain in the system: a large amount of "resources" are wasted. The "resources" mentioned here are actually treated as waste by villagers, which include not only organic substances, including abandoned limbs and chestnut shells in agricultural production, and food scraps and poultry feathers in poultry, etc., as well as inorganic substances, which include food packing, raw material packing of crops, etc.. When dealing with waste, the villagers cannot achieve the sorting of organic and inorganic substances, so that waste cannot be used efficiently, and a large number of "resources" are simply buried, even burned outdoors, resulting in air and dust pollution. Another kind of "waste of resources" is the waste of energy, first of all, the use of abandoned limbs for incineration is a very low rate of conversion. The waste of water resources is also a thorny problem. In the theory of system design, water resources are divided into several levels: drinking water, grey water (available water) and black water (the water that causes pollution), and during the daily life of the villagers there, a large number of water with the drinking water level is directly used for activities that do not require high water level water, which in itself is a waste of water resources.

\subsubsection{Environmental pollution factors}

A great deal of data tells us that there is a limit to the carrying capacity of the environment which can purify itself within a certain range. Through the analysis of the dietetic cultural system in Lifengjie, the author finds that a great deal of chemical fertilizer and chemical herbicides are the source of environmental pollution in its agricultural production, while chemical elements cannot be quickly degraded naturally, which move into the soil and water sources and enter the human body with the plant chain, and after accumulating to a certain amount, they can affect the health of the human body. Another source of pollution comes from the burning of plant stems, which produce large amounts of carbon dioxide gas and harmful gases that do not burn enough, in addition, when dust enters the respiratory tract of the human body, it can also cause damage to the human body. Therefore, in the subsequent systematic transformation, it is necessary to reduce the access of chemical fertilizers, and avoid the method of burning plants is used again in life and fertilization.

\subsection{Systemic}

In the recycled material recycling system, clean energy is used more often in residents' lives, for example, they mainly use electricity to control the temperature of the environment and to clean their daily lives, and compared with the burning of dried bamboo and dead branches, it can reduce a large number of harmful greenhouse gas emissions, and reduce dust pollution, thereby improving the living environment of residents and environmental benefits; in the use of detergents, the author advocates to reduce the use of chemical detergent and washing by physical methods or using biodegradable detergent, and residents can even make lemon and sodium bicarbonate detergent at home, thus reducing the chemical pollution to the base soil and water; in the water cycle, when the drinking water enters the diet, the residue of the solid residue after washing can be removed from the grey water, and the grey water can be used for the cleaning of the individual or the living environment to realize the hierarchical utilization of the water quality, finally, the black water which cannot be used directly can be introduced into the septic tank for purification, in addition, the air conditioning water when using air conditioning can also be used for environmental cleaning. Through the reconstruction of the water cycle of the residents' living system in Lifengjie, the author realizes the multi-level utilization of water resources, thus reducing the waste of water resources; and the residual organic matter in the residents' 
lives can be input into poultry breeding and renewable energy systems to achieve multi-level trophic stratification.

\subsubsection{Agricultural production system}

In the "food production" system in Lifengjie, rice planting and bamboo forest industry is the largest, and thus the use of water resources is also the most, therefore, rice planting and bamboo forest industry can be taken as the starting point, and water stratification can be used in the two quarter of spring and autumn, water can be used as a medium, and the nutrients in water can be layered and utilized at many levels, thus saving water resources and saving nutrients. The system flow to implement this idea is: Use the river water to irrigate paddy fields, remove evaporation water, and use the rest of the water for vegetable and chestnut farming, and grey water with nutrients is used in poultry farming and aquaculture, until the water contains harmful substances and turns into black water and concentrates on its water purification system, while the bamboo forest industry uses natural rainfall to irrigate the surface water and consciously guide the pond for aquaculture. Farmers can arrange crops to select crops according to the information in their solar terms, so as to ensure every solar term can provide the appropriate solar term food for the dietary system in the Lifengjie scenic spots. In its agricultural production system, the surplus of rice, straw and other organic materials are no longer converted to fertilizer by burning, but are concentrated in its renewable energy system, and then compost through biogas digesters, while producing biological fertilizer, the biogas provided can be used for the living system of the residents and the solar diet and commercial system. In addition, the remaining organic matter such as rice can be used as a substitute for the chemical nutrients for poultry farming and aquaculture. At the same time, some of the raw materials in the agricultural production system can be kept for breeding and returned to the system.

\subsubsection{Commercial system}

In the past, because it was not developed for a large scale, it did not have commercial diet. With the completion of the twenty-four solar tourism development, the solar commercial system will become a major source of income for residents in Lifengjie and an important component of its system transformation. Sources of ingredients for the solar business system is partly from its own solar term ingredients, including rice, seasonal vegetables, spring bamboo shoots, wild mushroom etc., the other source is from the featured food around Liyang, such as Tianmu Lake fish head, Liyang white tea and all kinds of fruits and so on. The black-water produced in the throttle restaurant is introduced into the integrated water purification system of Lifengjie. The organic residue in the solar energy restaurant can be introduced into the poultry breeding program to achieve multi-level trophic stratification.

\subsubsection{Water treatment system}

In the past, it did not have a comprehensive water purification system. In the future, the use of water resources, which had not been sufficient, would have been more intense as its population and visitor population increased, therefore, under the premise of ensuring the rational use of water resources, which is of great significance to carry out the complete planning and rational use of water resources. The future water treatment system will transform the grey water from the "food production" system, the living system of residents and the commercial diet of the solar system into recycled water and return it to the system through foam separation, septic tank solid-liquid separation, water purification, plant filtration and other stages, and the solid waste separated from the septic tank can enter the renewable energy system and can be converted into bio fertilizer and clean energy.

\subsubsection{Renewable energy system}


Now, some families have methane pools, in the new system, the large-scale renewable energy system will be built with Lifengjie as a whole, collect solid waste that can be converted into biogas and organic fertilizer, and thus the organic fertilizer produced by the renewable energy system flows to the agricultural production system, and biogas can be used for the food and cooking of the residents' living system and the solar business system. The designed material flow system is showed in Figure. 4.

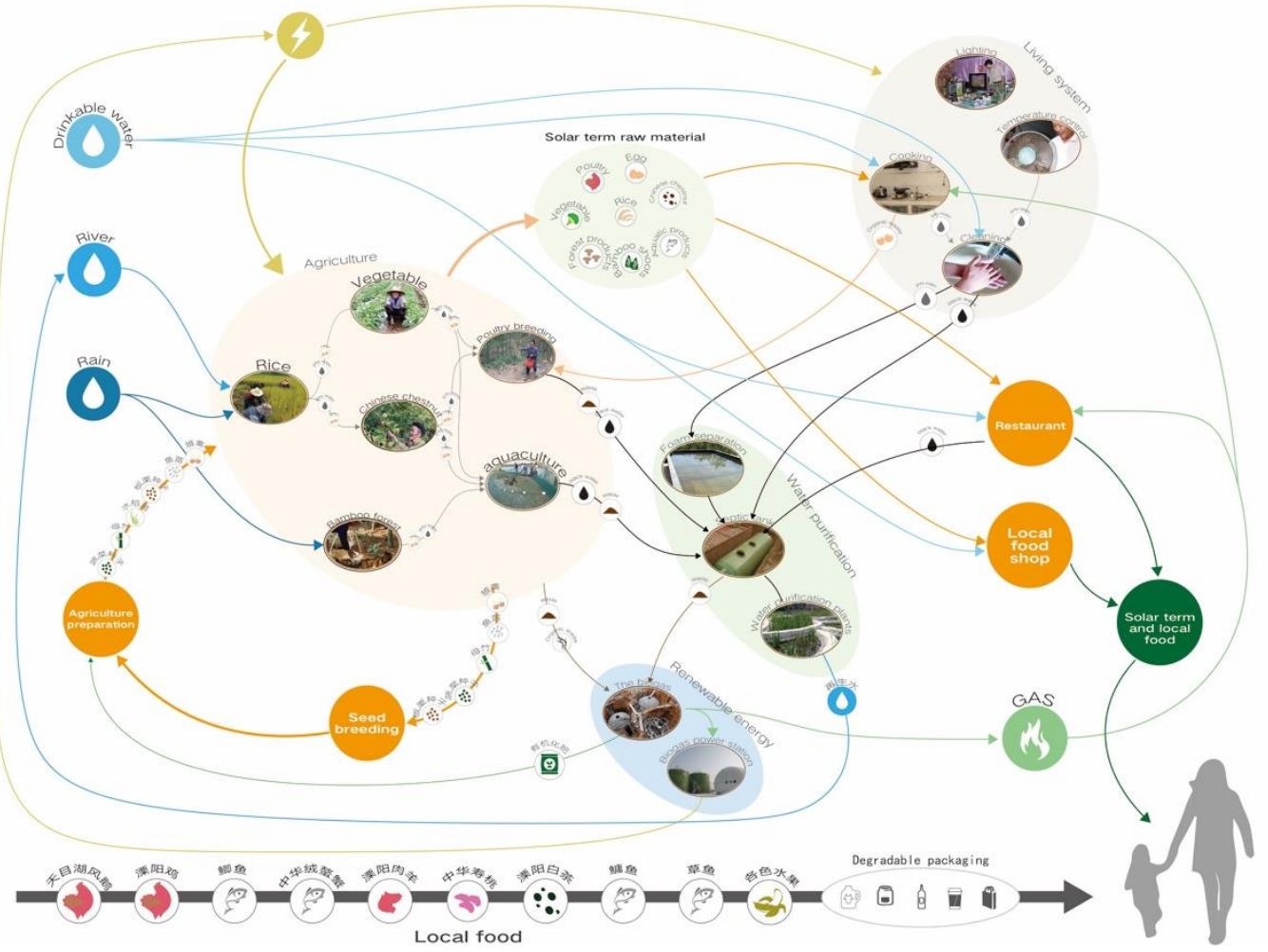

Figure 4. Solar terms ecosystem

On the basis of subsystem reconfiguration, the author combs the relations among subsystems, in general, when reconstructing the circulation of agricultural production related substances from the perspective of the dietary culture of the solar terms, the concept of solar energy ingredients is emphasized, and a new material cycle is built around the solar energy ingredients, and in the new system, the solar feed is provided by the agricultural production system and flows into the solar energy commercial system and the living system of the residents. As the material basis of constructing the system of solar term food culture, other material circulation should follow the concept of twentyfour solar terms, which adopts measures suiting local conditions, and does farm work in the right season, and study modern ecological agriculture and recycle agriculture to plan, so as to reduce the material needs of the system, and to produce less waste of system materials, and thus improve the overall efficiency of the use of resources.

Then we start each subsystem in the system, and finally get its material flow of dietetic culture of the twenty-four solar terms, and system material cycle diagram. From the perspective of tourists, they buy food ingredients from its own seasonal ingredients, which mainly include rice, vegetables, chestnut, spring bamboo shoots, wild mushroom products etc., on the other hand, it is provided by the nearby areas, such as Tianmu Lake fish head, seasonal fruits, meat, Liyang white tea and other products. The support system for self-produced seasonal ingredients can not only ensure the output of seasonal ingredients, but also provide seasonal agricultural tourism experience, and because the 
system meets the requirements of circular agriculture, it is also an important part of its natural environment.

\section{Conclusion}

\subsection{Economic performance}

On the whole, we re-plan Lifengjie based on the diet of the twenty-four solar terms, and add a new commercial diet to it, changes in the traditional population can only rely on the wholesale of crops to the status of crop wholesalers, thus raising the possibility of increasing income for residents. And in accordance with the twenty-four solar terms, their production, festivals, food habits can be regarded as a tourist feature to attract more tourists, and more tourists will therefore bring more economic benefits.

\subsection{Environmental benefit}

The concept of adopting measures suiting local conditions and twenty-four solar term in local conditions, and doing farm work in the right season can serve as a guiding principle in the reconstruction of rural material flow system, and based on the idealized transformation of the twentyfour solar terms in Lifengjie, in the new system, nutrients, energy and water resources can be classified and utilized to improve the utilization of resources, establish a water purification system and a renewable energy system, improve the recycling efficiency of waste and black water and improve the overall efficiency of the system; on the other hand, the new system opposes the use of chemical cleaning agents, fertilizers and so on, and advocate the use of biological detergents, biological fertilizer, physical, insect and other means to reduce environmental pollution sources into the system. Ideally, Lifengjie can benefit from the environment.

\subsection{Cultural benefit}

In the survey, the author can appreciate the sense of pride in the local culinary culture, in this topic, the author has long established the design principle of enriching the connotation of solar terms on the basis of protecting local culture, and in the design, based on the twenty-four solar terms, the author combs the connotation of the base diet culture, and links each element with the solar terms, change its eating habits from the concept, and thus strengthen the soft power of Liyang food culture.

\section{References}

1. P. Cristiana, P. Migliorini, F. Sottile, Eur. Ecology and Society. J. E 19, 4 (2014)

2. G. Pauli, The Blue Economy (Academic Foundation, 2016)

3. C. Peano, N. Tecco, E. dasero, V. girgenti, F. Sottile, Eur. Sustainability. J. E 7, 6741 (2015)

4. L. Bistagnino, System Design: designing the productive and environmental sustainability(Slow food, 2011)

5. F. Capra, The Web of Life (Anchor, 1997) 